\title{
ENVELHECER COM VIH/SIDA: UM CONTRIBUTO PARA A ANÁLISE DAS POLÍTICAS DE SAÚDE E DIREITOS NA UNIÃO EUROPEIA
}

\section{AGING WITH HIV/AIDS: A CONTRIBUTION TO THE ANALYSIS OF HEALTH POLICIES AND RIGHTS IN THE EUROPEAN UNION}

\author{
Fernando Bessa Ribeiro* \\ Octávio Sacramento \\ Marta Maia $^{* * *}$
}

\begin{abstract}
RESUMO
Acrescente cronicidade do HIV $/ \mathrm{sida}^{1}$ conduziu a um aumento significativo do número de doentes com mais de cinquenta anos, fazendo com que o seu envelhecimento adquira relevância social e política. Tendo como contexto geopolítico a Europa e explorando dados estatísticos e documentos, elegemos como questão principal a análise das políticas de prevenção dirigidas para as pessoas mais idosas e das possibilidades do direito, através do reforço do quadro legal, para a proteção dos seus interesses. Num primeiro momento, a análise circunscreve-se ao mapeamento estatístico e à compreensão da crescente prevalência da epidemia entre as pessoas idosas, bem como das suas principais consequências e desafios. De seguida, avaliamse as políticas públicas implementadas para enquadrar a gestão das interseções entre a enfermidade e o envelhecimento. Das conclusões releva-se a necessidade de fundar as práticas de cuidado e proteção dos idosos seropositivos num quadro de direitos fundamentais, a implementar através de políticas públicas específicas, à semelhança do que já acontece para outros grupos populacionais considerados como particularmente sujeitos a múltiplas vulnerabilidades e constrangimentos.
\end{abstract}

Palavras-chave: VIH/sida; Envelhecimento; Europa; Políticas e direitos.

\begin{abstract}
Considering the statistical, social and epidemiological relevance of people over 50 years living with HIV/AIDS, the article discusses the disease manifestations in the framework of aging in Europe and the requirements arising in terms of public policies and rights promotion. The analysis is focused, first of all, on the statistical mapping and understanding of the growing prevalence of the epidemic among the elderly and its main consequences and challenges. At a later stage, the public policies that have been implemented to guide the management of the intersections between HIV/AIDS and aging are evaluated, leaving a critical note on the need to base the care and protection practices of the seropositive elderly in a rights framework, as is already the case for other groups considered to be particularly susceptible to several vulnerabilities and constraints.
\end{abstract}

Keywords: HIV/AIDS; Aging; Europe; Policies and rights.

\footnotetext{
"Universidade do Minho, Portugal. fbessa@ics.uminho.pt

${ }^{*}$ Universidade de Trás-os-Montes e Alto Douro, Portugal. octavsac@utad.pt

*** Filiação institucional.maia_marta@hotmail.com

${ }^{1}$ Vírus da imunodeficiência humana/síndroma da imunodeficiência adquirida (VIH/sida em vez de HIV/aids, utilizada no Brasil). Fazemos notar que escrevemos segundo o novo acordo ortográfico. Apesar de ter aproximado os modos de escrever português em Portugal e no Brasil, subsistem diferenças que o leitor facilmente notará.
} 


\section{INTRODUÇÃO}

Em boa medida devido ao verificado na fase inicial da doença, atingindo sobretudo homens jovens, a infeção pelo VIH/sida tem sido considerada uma epidemia que afeta sobretudo adultos abaixo dos quarenta anos, parecendo inesperada a sua prevalência significativa em pessoas acima dos cinquenta anos (Brooks et al., 2012). A progressiva cronicidade da doença decorrente da eficácia das marcantes inovações terapêuticas de finais da década de 1990 (Camoni et al., 2014) permitiu às pessoas infetadas continuarem vivas, envelhecendo-as. É preciso ainda ter em conta que os mais idosos não deixam de estar sujeitos a fatores de risco face ao VIH, apesar de poderem estar menos informados e conscientes disso, como já na década passada observaram, entre outros, Nguyen; Holodniy, 2008). Em concreto, a sua atividade sexual constitui, como sublinha Banens (2016), uma questão da maior relevância, nomeadamente em termos da disseminação da infeção, devido à eventual continuação de comportamentos de risco por desconhecimento ou insuficiente sensibilização para práticas sexuais seguras. Sendo uma epidemia sem fronteiras etárias, ninguém fica à margem, pelo que, potencialmente, "todos se encontram em risco" (Piot; Cravero, 2007, p. 179).

De certo modo, a infeção pelo VIH/sida entre as pessoas acima dos cinquenta anos é ainda uma "uma história inexplorada" (Schmid et al., 2009), nomeadamente no que diz respeito aos efeitos produzidos no envelhecimento físico dos que vivem com ela (Wallach, 2012). Atendendo ao carácter crescentemente crónico da infeção pelo VIH/sida, uma parte significativa dos indivíduos por ela atingidos irá também envelhecer e será, incluindo do ponto de vista estatístico, bastante representativa. Sendo o envelhecimento um fenómeno que não depende apenas de fatores biológicos, mas também (e sobretudo) de fatores sociais, ${ }^{2}$ a infeção do VIH/sida na população mais idosa coloca questões relevantes e desafios substanciais às políticas de saúde

\footnotetext{
${ }^{2}$ Embora possa ser entendido como um lugar-comum sociológico, não será descabido sublinhar, considerando o crescimento acentuado, nas últimas décadas, das desigualdades sociais nas sociedades contemporâneas (Piketty, 2013), que não vivemos todos nas mesmas condições sociais e económicas. Daqui decorre que não padecemos das mesmas doenças nem envelhecemos da mesma forma, sendo o envelhecimento e as patologias que nos atingem em larga medida consequência da nossa posição na estrutura social e estilo de vida a ela associada. Ou seja, a classe social é também um elemento-chave para se compreender os efeitos da doença nos indivíduos, incluindo no domínio do VIH/sida (cf., entre outros, Ribeiro; Sacramento, 2012; Hohmann; Olimova, 2016; Bletzer, 2016; O’Grady, 2016).
}

pública na União Europeia (UE). É o caso do financiamento das despesas com este grupo de pessoas e o respetivo impacto nos orçamentos para a saúde. Ao mesmo tempo, esta intersecção entre VIH/sida e envelhecimento suscita preocupações adicionais quanto ao respeito de direitos fundamentais em relação às pessoas idosas. Sabendo-se que não existem instrumentos legais transnacionais de grande escala, a nível da Organização das Nações Unidas (ONU) ou sequer da UE, especificamente vocacionados para tal, daqui decorre que "os direitos dos idosos são muitas vezes ignorados ou até mesmo negados, o que demonstra que o enquadramento legal internacional vigente não garante aos idosos os seus direitos humanos". (Miller, 2010, p. 347).

Com base na consulta de fontes documentais e bibliográficas, escrutinam-se os principais dados estatísticos disponíveis sobre a infeção, sempre com uma especial atenção à situação europeia. Em seguida, são analisadas as políticas públicas e os direitos na Europa no que diz respeito à saúde e ao envelhecimento, prestando aqui especial atenção à situação das pessoas com mais de cinquenta anos portadoras da infeção pelo VIH/sida, geralmente identificadas como " $50+$ ".

Sendo a UE uma "região" no qual predominam políticas de saúde ancoradas em sistemas públicos robustos, largamente dominadas por financiamento e até provisão públicas, no quadro do Estadoprovidência, as políticas no campo da infeção pelo $\mathrm{VIH} /$ sida constituem um objeto relevante para a análise sociológica e política, podendo contribuir para um melhor conhecimento e ação em outras geografias. Assim, a partir da análise de documentos e normativas que contribuem para definir e regular a intervenção pública neste domínio, o objetivo do nosso ensaio é discutir as competências e recursos das instituições europeias supranacionais no campo da luta conta a infeção pelo VIH/sida, identificando-se não só as suas insuficiências e ausências, mas também os aspetos positivos e concomitantes sucessos. Tendo como questão principal examinar as políticas de prevenção dirigidas às pessoas mais idosas, será que estas acabam diluídas no contexto geral da luta contra a infeção, em boa medida porque as instituições envolvidas na prevenção olham para este grupo de doentes como indivíduos sem grande atividade sexual, logo não representando risco significativo em 
termos de prevenção? Responder à questão implica analisar a ação a nível europeu no combate a esta epidemia que, não obstante a sua cronicidade, continua a ser excecional (Whiteside, 2008, p. 124), pelo que as soluções têm de ser, como já o defendemos em texto anterior (Ribeiro; Sacramento, 2015), também excecionais, mobilizando os recursos e as competências técnicas no domínio da intervenção social (Sacramento; Ribeiro; MAIA, 2016).

\section{ENVELHECER COM VIH/SIDA NA EUROPA}

Desde que o primeiro medicamento, o Azidothymidine - também designado por Zidovudine ou, simplesmente e mais comummente pela sua abreviatura, Azt - começou a ser disponibilizado aos doentes em 1990, muitas mudanças ocorreram no tratamento da infeção pelo VIH/sida. As sucessivas inovações terapêuticas de alta eficácia permitiram progressos significativos no controlo da infeção pelo $\mathrm{VIH} /$ sida $^{3}$ De doença mortal foi passando a doença crónica, tendo sido definido pela comunidade internacional em 2015 o objetivo de terminar com a epidemia do VIH/sida enquanto ameaça à saúde pública em 2030 (WHO, 2016). Ora, para o conseguir haverá que reduzir severamente as infeções pela via sexual entre adultos, agindo sobre um cenário relativamente inquietante: a persistência, nos últimos anos, de novas infeções neste grupo. Apesar de estarmos longe dos cenários terríveis dos primeiros anos da epidemia, quando a completa ausência de recursos terapêuticos produziu, por um breve período de tempo, uma certa unificação do mundo ao colocar qualquer indivíduo, rico ou pobre, na mesma situação perante a infeção (Bastos, 2002, p. 21), as mortes por VIH/ sida continuam a mostrar que, inclusive na Europa, continuamos largamente aquém de um dos objetivos definidos pela Onusida: o desaparecimento da mortalidade provocada por esta doença (WHO, 2016). Com 36.9 milhões de pessoas a viver com a infeção, com entre 670.000 e 1.3 milhões a morrer de causas relacionadas com ela em 2017, sendo o valor médio estimado de 940.000, traduzindo-se numa redução de cerca de 34\% face a 2010 (Unaids DATA, 2018), a taxa de mortalidade por $\mathrm{VIH} /$ sida na UE ascendeu em 2016 a 0.8 por 100.000 habitantes (ECDC/WHO Regional Office for Europe, 2017).

Perscrutando mais alguns dados sobre a situação da doença a nível internacional, 21,7 milhões estão em tratamento - o que perfaz quase $60 \%$ do total de infetados com acesso a medicação - e cerca de 1.8 milhões foram infetados em 2017. Muito relevante, desde 2010 que o número de novas infeções entre adultos permanece estável. Não obstante este manifesto fracasso, atendendo ao objetivo da redução a zero de novas infeções, o número de mortes relacionadas com a infeção reduziu-se em cerca de $45 \%$ desde o seu pico alcançado em 2005 (UNAIDS DATA, 2018). Constatando a relevância da infeção entre os adultos mais idosos, em 2013 a Onusida apresentou um suplemento ao relatório geral (Unaids, 2013) onde destaca uma tendência cada vez mais significativa na epidemia global da infeção pelo VIH/sida: o aumento do número de indivíduos idosos que vivem com a doença, representando cerca de $1 / 3$ do total de doentes na Europa ocidental e na América do Norte. Os dados mais recentes, referentes a 2016 (Unaids, 2016a), mostram que, no mundo, $17 \%$ dos indivíduos seropositivos com quinze ou mais anos têm mais de cinquenta anos, sendo que nos países pobres já representam cerca de $15 \%$ do total de adultos.

Recorrendo aos relatórios de 2016 e 2017 do ECDC/WHO Regional Office for Europe, nesta área geográfica foram 849 as mortes em 2016 relacionados com a infeção pelo VIH/sida. Trata-se de um valor positivo impressivo. Apesar de ainda estar longe do objetivo "zero mortes" da Onusida, o resultado é muito meritório quando comparado com o valor de 2007, ano em que ocorreram 2927 (2928 considerando a Noruega) mortes, Gráfico 1. Mortes por causas relacionadas com a infeção pelo VIH/sida na UE/Espaço Económico Europeu). Uma tendência preocupante, exprimindo uma situação de grande resiliência, é a das novas infeções, mostrando que se continua muito aquém da concretização de outro grande objetivo fixado pela Onusida, o da eliminação do contágio.

${ }^{3}$ Estes múltiplos recursos são denominados por terapêutica antirretroviral de combinação (TARC), sendo também comum a utilização da designação em língua inglesa Highly Active Anti-Retroviral Therapy (HAART). 
Gráfico 1: Mortes por causas relacionadas com a infeção pelo VIH/sida na UE/Espaço Económico Europeu

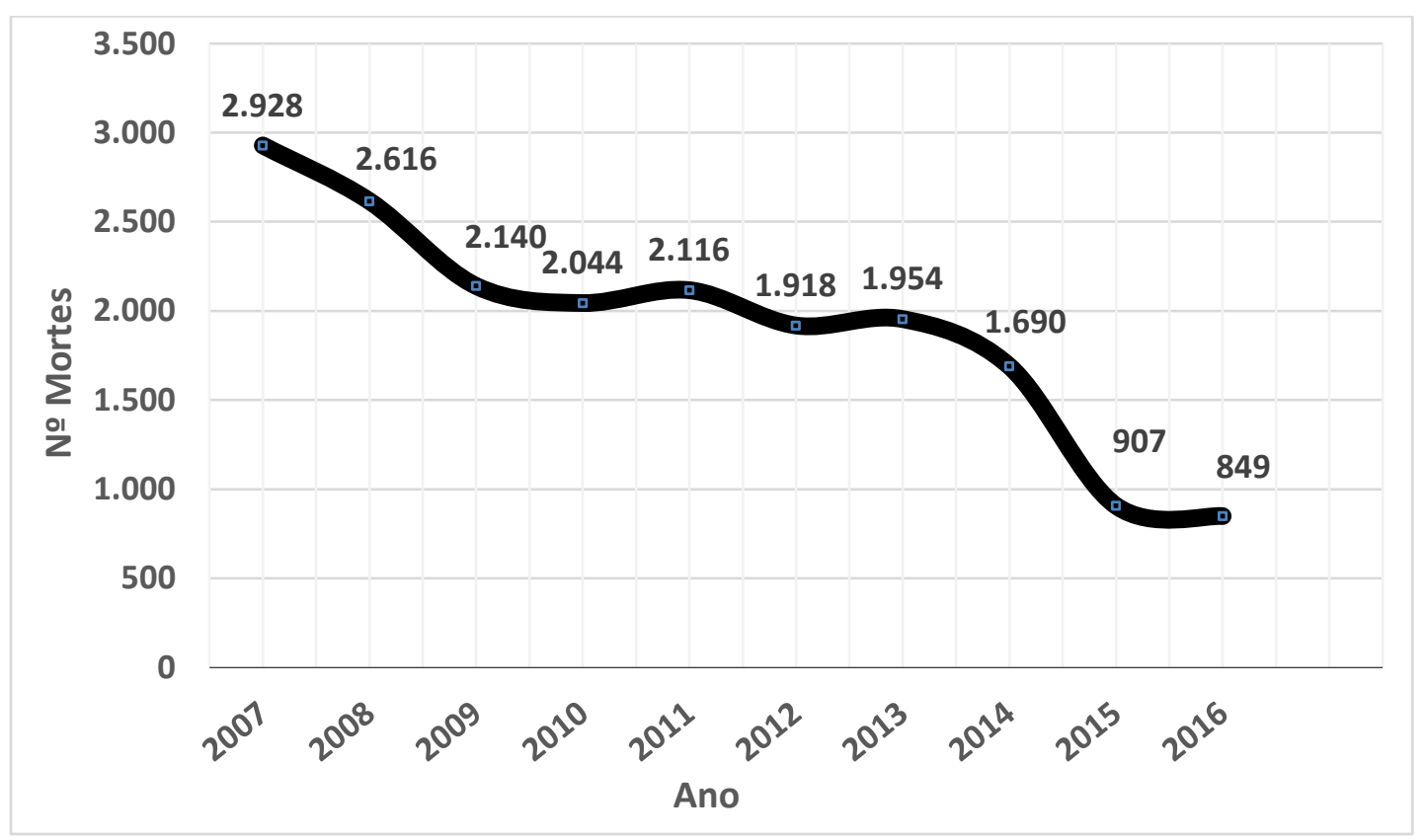

Fonte: ECDC/WHO Regional Office for Europe (2017) (adaptado).

Os indivíduos com cinquenta ou mais anos representam 39\% da população da UE/EEE infetada e $18,5 \%$ dos novos diagnósticos de VIH. Em seis países (Finlândia, França, Malta, Países Baixos, Noruega e Portugal), este grupo de indivíduos representou mais de $20 \%$ dos casos diagnosticados em 2016. Não menos relevante, a percentagem de indivíduos neste grupo com diagnóstico tardio é muito elevada - tomando como referência as 350 células CD4 por $\mathrm{mm}^{3}$ -, atingindo $63 \%,{ }^{4}$ quando a média geral fica pouco acima dos $50 \% .^{5}$

Equacionando as causas da crescente prevalência da infeção pelo VIH/sida entre os mais velhos, os responsáveis da Unaids pelo documento de 2013 acima mencionado argumentam que o aging da epidemia se deve fundamentalmente a três fatores: $(i)$ a eficácia da terapia antirretroviral, permitindo diminuir a mortalidade precoce; (ii) a diminuição da incidência da infeção entre os jovens adultos; (iii) maior exposição dos indivíduos com mais de cinquenta anos a

\footnotetext{
${ }^{4}$ Cluster of Differentiation 4 (Agrupamento de Diferenciação 4) é um conjunto de moléculas presentes na superfície celular que permite identificar e diferenciar diversos tipos de células.

${ }^{5}$ Este diagnóstico tardio representa uma situação de risco acrescido para estes indivíduos, revelando os testes uma contagem de células CD4 sistematicamente inferior quando comparada com indivíduos mais jovens em idêntica situação, pelo que a progressão da infeção pelo VIH/sida é mais rápida em idades mais tardias (Nguyen, Holodniy, 2008).
}

comportamentos de risco, devido a menor informação e sensibilização. Em linha com este diagnóstico, outros autores argumentam que a crescente prevalência da infeção entre as pessoas idosas, sobretudo nos países ocidentais, acompanha o "inverno demográfico" desses mesmos países (Dumont, 2007), ${ }^{6}$ explicando-se em função de vários fatores como: $(i)$ a cronicidade da infeção, devido à introdução das TARC em finais da década de 1990; (ii) a melhoria generalizada da saúde entre os idosos, favorecendo a manutenção da sua atividade sexual até mais tarde; (iii) o incremento das taxas de divórcio entre as pessoas com cinquenta e mais anos, criando condições potenciais para uma maior variabilidade de parceiros sexuais; (iv) a predisposição para uma menor utilização do preservativo, sobretudo por ser percebido, amiúde, mais como um meio de contraceção do que de profilaxia; (v) a "crença" no VIH como um problema dos jovens (Wallach et al., 2013, p. 11).

\footnotetext{
${ }^{6}$ Sendo um conceito expressivo para caracterizar o envelhecimento, o "inverno demográfico" exige alguma precaução na sua utilização, sobretudo se interpretado de uma forma muito negativa. Fazendo o necessário contraponto, o envelhecimento da população a nível global, especialmente notório na maior parte dos países ocidentais, é consequência dos avanços positivos no campo da habitação e saúde pública, ocorrendo num mundo ainda muito pressionado pelo crescimento demográfico. Assim, as eventuais dificuldades suscitadas por tal "inverno" terão de ser analisadas considerando o modo como se organizam as políticas públicas, a economia e a distribuição da riqueza nos países com maior percentagem de população idosa.
} 
Tendo a região europeia da Organização Mundial de Saúde (OMS) superado, ${ }^{7}$ no final de 2016, os 2.1 milhões de pessoas diagnosticadas cumulativamente com o VIH/sida, nesse ano foram diagnosticados mais de 160.453 novos casos, o valor mais alto desde que existem dados estatísticos sobre a doença. Para a UE/EEE os novos casos perfazem quase 30.000 indivíduos, com uma taxa inferior a 6 por 100.000 habitantes, algo que já não se verificava desde 2003, com uma forte prevalência de infeção entre os homens que fazem sexo com homens (HSM), com 40,1\% e dos heterossexuais, com 32,3\%, conforme o Quadro 1). Daqui decorre que continua sem se verificar uma redução substancial de novos casos de infeção na UE/ EEE, não obstante, como sublinha o relatório citado, as medidas de prevenção implementadas.

Quadro 1: Dados gerais sobre a infeção pelo VIH/sida na EU/EEE em 2016

\begin{tabular}{|l|l|}
\hline NOVOS DIAGNÓSTICOS & $\mathbf{2 9 4 4 4}$ \\
\hline Rácio por 100000 habitantes & 5.9 \\
\hline Percentagem entre 15-24 anos & $10.6 \%$ \\
\hline Percentagem 50+ & $18.5 \%$ \\
\hline Relação masculino-feminino & 3.2 \\
\hline $\begin{array}{l}\text { Percentagem de novos diagnósticos tardios } \\
(350 \text { células CD4 por mm })^{3}\end{array}$ & $47.7 \%$ \\
\hline MODO DE TRANSMISSÃO & \\
\hline Sexo entre homens & $40.1 \%$ \\
\hline Heterossexual & $32.3 \%$ \\
\hline Utilizadores de drogas injetáveis & $3.6 \%$ \\
\hline Transmissão mãe-recém nascido & $0.5 \%$ \\
\hline Desconhecido & $23.5 \%$ \\
\hline
\end{tabular}

Fonte: ECDC/WHO Regional Office for Europe (2017) (adaptado).

Por sua vez, os dados revelam que os homens jovens - grupos entre 25 e 39 anos - continuam a ser os mais atingidos, não sendo de modo algum desprezável estatisticamente o peso da contaminação entre os mais velhos do sexo masculino, sobretudo no grupo dos 40 aos 49 anos (Gráfico 2).

Gráfico 2: Idade e género de novos casos de VIH/sida diagnosticados por 100.000 habitantes na UE/EEE em 2015 (n=29.639)

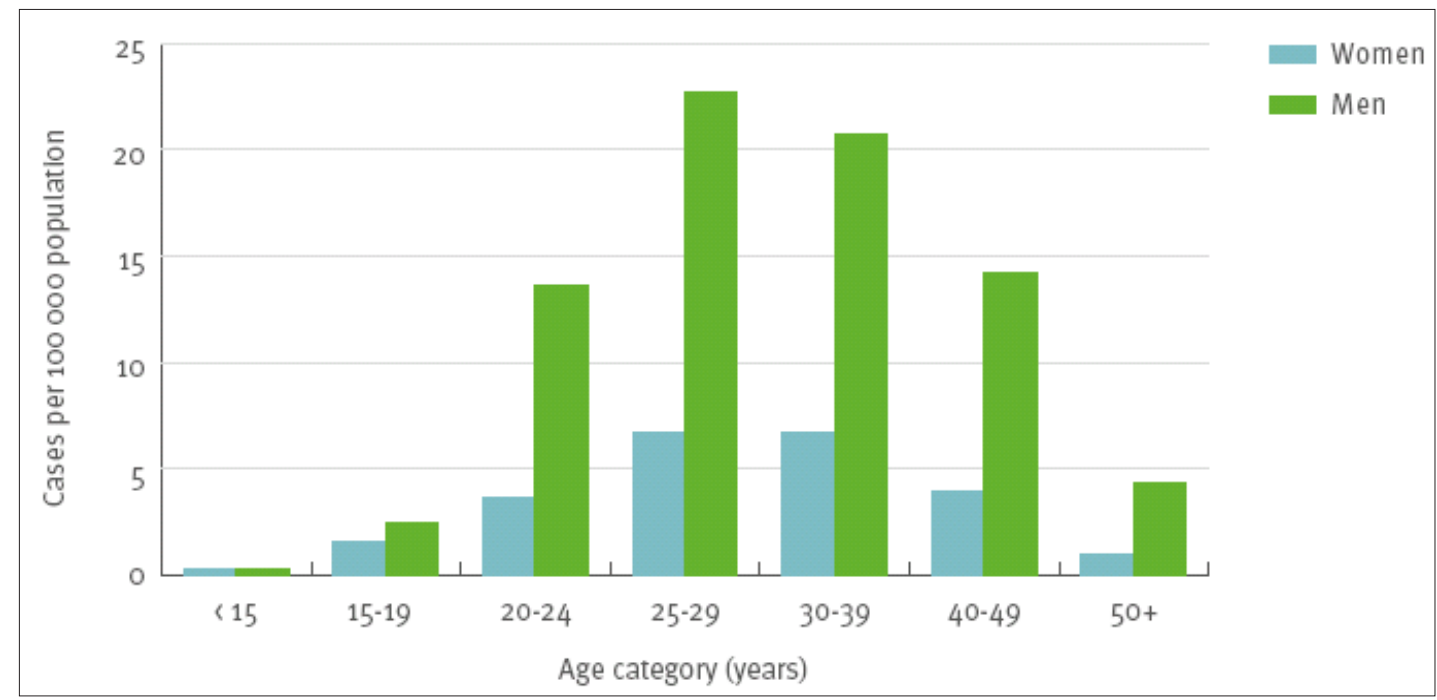

Fonte: ECDC/WHO Regional Office for Europe (2016).

\footnotetext{
${ }^{7}$ A região europeia da OMS é constituída por 53 países, cobrindo uma área geográfica que se estende desde Portugal, no Atlântico, até à Rússia no Pacífico. Nela estão incluídos todos os estados-membros da UE (WHO, 2018).
} 
Entre os mais velhos, as formas mais prevalecentes de contaminação são o contacto heterossexual, particularmente relevante nos indivíduos com mais de cinquenta anos, representando $20 \%$ do total das infeções deste tipo, e o contacto homossexual (Gráfico 3). Sendo manifestos os riscos a que este grupo está sujeito, impõe-se uma especial atenção no domínio da prevenção orientada para a redução de comportamentos de risco dos indivíduos. Observe-se que as pessoas com mais de cinquenta anos não constituem um grupo homogéneo, antes sendo atravessado por diferenças de género, de capital económico, escolar e organizacional, de nacionalidade e orientação sexual, bem como de distintos comportamentos e consumos $-v \cdot g$., álcool, estupefacientes por via injetável - que concorrem para que encontremos múltiplas situações. Seguindo a formulação proposta por Lazarus e Nielsen (2010), é possível proceder a uma grande divisão neste grupo: $(i)$ a parcela dos indivíduos que se infetaram ainda jovens, sobreviventes devido ao aparecimento de fármacos TARC, hoje com mais de cinquenta anos; e (ii) a parcela dos indivíduos que se infetaram em idade já mais avançada da vida, a quem é diagnosticada a doença, em regra, numa fase mais tardia.

Gráfico 3: Novos casos de VIH/sida diagnosticados por grupo de idade e modo de transmissão na UE/EEE em 2015 (n=23.417)

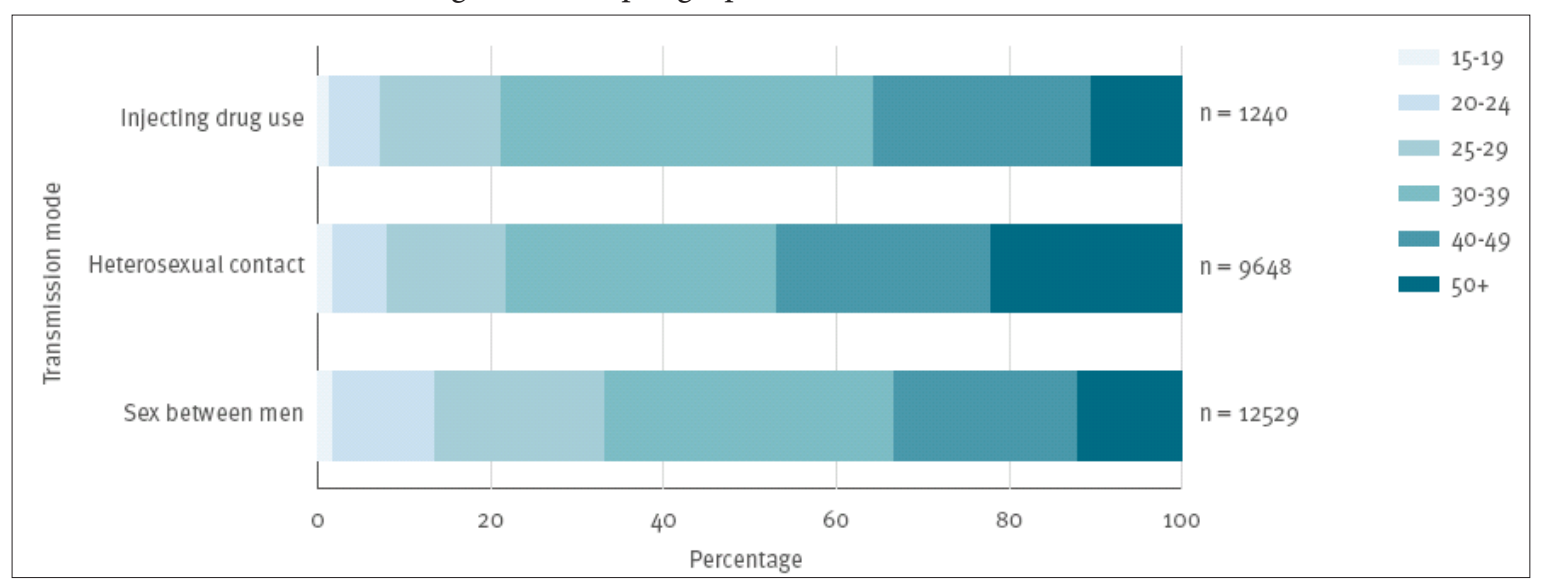

Fonte: ECDC/WHO Regional Office for Europe (2016)

A infeção pelo VIH/sida continua a ser, simultaneamente, um desafio e uma preocupação maior para os governos e suas políticas no campo da saúde. Os dados mais recentes mostram que o número de novos casos permaneceu relativamente estável na UE/EEE, apesar do aumento em 2015 face ao ano anterior, continuando os estados a dedicar uma atenção especial à prevenção e controlo da infeção. Um dos exemplos relevantes deste empenhamento é o aumento em 78\% do número de testes na UE entre 2006 e 2016, passando de 12.578 .889 para 22448461 .

Encerrando a análise dos dados estatísticos, e como foi já aludido, o número de mortes decorrentes da infeção reduziu-se significativamente ao longo dos últimos dez anos, em especial entre 2013 e 2015. Não podendo ser separada da robustez dos sistemas de saúde e das políticas públicas que o suportam, esta situação está também relacionada com os efeitos decorrentes do avanço nas terapêuticas antirretrovíricas. Porém, apesar dos significativos progressos biomédicos nas duas últimas décadas e da relativa normalização do VIH/sida, nomeadamente nos países ocidentais, como mais um dos muitos problemas de saúde pública (Whiteside; Smith, 2009), ${ }^{8}$ a infeção continua a suscitar inúmeros desafios sanitários e sociais, muito em especial para as pessoas idosas. Estas debatem-se já com inúmeros problemas de saúde crónicos e, do ponto de vista social, nem sempre beneficiam de apoios adequados e consistentes:

Embora a aceitação do HIV/AIDS como uma condição crônica para adultos mais velhos possa ajudar a melhorar seu tratamento médico e suas percepções da expectativa de vida, os adultos mais velhos com HIV/AIDS ainda enfrentam muitos obstáculos a serem superados. O envelhecimento com HIV/AIDS

\footnotetext{
${ }^{8}$ Tratar-se-á, porém, de uma normalização relativa. Mesmo deixando de ser mortal, a infeção VIH/sida continuará ainda por muitos anos a ser uma doença "excecional", considerando, entre outras razões, a forma como se transmite e a estigmatização decorrente da censura social sobre determinadas práticas e estilos de vida entendidos como propiciadores da sua disseminação (Moyer; Hardon, 2014).
} 
é mais difícil do que o envelhecimento com condições crônicas relacionadas à idade (como doenças cardíacas e diabetes), associando estigma social e o subsequente isolamento social, e dificuldades relacionadas com a adesão à medicação e interações medicamentosas (Pelt, 2012, p. 28).

Quer isto dizer que os mais idosos, independentemente da idade em que se infetaram, enfrentam uma forte vulnerabilidade que pode refletir-se mesmo em períodos de sobrevida mais curtos. Como sugerem os dados apresentados, a demora na deteção do VIH entre as pessoas $50+$ está relacionada com avaliações incorretas por parte dos profissionais de saúde. Não raro, estes consideram, por um lado, que os sintomas indicam outras patologias próprias dos mais velhos, por outro olham para esta população como não estando sujeita a uma situação de risco elevado de infeção (Cardoso et al., 2013, p. 464). Esta última razão decorre da "invisibilidade da sexualidade no idoso" (Alencar; Ciosak, 2016, p. 1142), uma tendência social generalizada que conduz os profissionais de saúde a naturalizar as pessoas mais velhas como assexuadas e, portanto, a negligenciar a sua vida sexual e os respetivos impactos em termos de saúde pública.

Inseparável dos problemas e fragilidades produzidas pelo aumento do risco de doença e o desgaste operado no corpo pelo decorrer dos anos de vida, a vulnerabilidade é também agravada pela experiência da doença crónica. Muitas vezes incapacitante, no caso da infeção pelo VIH/sida associam-se os constrangimentos no relacionamento social. Os seus efeitos atingem em especial os mais pobres, nomeadamente em situação de aposentação (Craveiro, 2012). Esta representa um período da vida em que, por efeito combinado da redução de rendimentos e aumento das despesas com saúde, com destaque para as relacionadas com os medicamentos, os indivíduos ficam sujeitos a formas acentuadas de privação material e de dependência. Também nesta doença, o género não é uma variável neutra, devendo ser considerado na medida em que os efeitos sociais da doença não se distribuem de forma semelhante entre homens e mulheres (Mèndes-Leite, 2015). Daqui decorre que as políticas públicas no campo da saúde desempenham um papel fundamental na definição e implementação de respostas adequadas a estes indivíduos idosos. Fazendo parte dos grupos sociais mais vulneráveis, esta situação não promove a proteção nem a adesão terapêutica e demais respostas socio-sanitárias à infeção.

\section{POLÍTICAS E DIREITOS PARA AS PESSOAS 50+ NA EUROPA}

A saúde é uma das áreas de ação política que continua a ser da responsabilidade dos estados-membros da UE. Porém, conforme o fixado na alínea k) do art. $^{\circ} 4^{\circ}$ e desenvolvido no art. $^{\circ} 168^{\circ}$ do Tratado sobre o Funcionamento da União Europeia (TFUE), os problemas relacionados com a saúde pública constituem um domínio de competências partilhadas entre a UE e os estados-membros. Assim, é aos governos nacionais que compete organizar e mobilizar os recursos necessários para a prestação de cuidados de saúde aos cidadãos, situação que não deixa, como acontece com muitas outras, de produzir severas diferenças entre os cidadãos europeus decorrentes das desigualdades estruturais que caracterizam a UE. ${ }^{9}$ Já à UE compete, no respeito pelo princípio da subsidiariedade, ${ }^{10}$ apoiar os estados-membros na concretização de objetivos-comuns, na organização de economias de escala por via da partilha de recursos e na resposta a desafios comuns como as doenças crónicas ou as pandemias (European Committee of the Regions, 2018; UNIÃO EUROPEIA, 2018).

Observando os documentos mais recentes, no Terceiro Programa de Saúde 2014-2020¹1, a UE procura continuar a promover a saúde a nível europeu, nomeadamente através da cooperação entre os estados-membros, em linha com o já fixado nos programas anteriores. Em concreto, pretende-se que o programa

\footnotetext{
${ }^{9}$ Podendo a desigualdade ser medida e notada a partir de diferentes critérios, o gasto em saúde per capita é relevante da situação em que se encontra a UE neste domínio fundamental para a vida dos cidadãos europeus. Enquanto (para 2015, ou ano mais próximo), a França gastou 3.342€ e a Alemanha $4.003 €$, já Portugal ou a Espanha gastaram em saúde, respetivamente, 1.967€ e $2.366 €$. Se observarmos os países mais pobres da UE, são vários os que gastam em torno dos $1.000 €$ per capita, como a Bulgária (1.108€), a Letónia (1.030€) e a Roménia (816€) (cf. OECD/EU, 2016).

${ }^{10} \mathrm{O}$ princípio da subsidiariedade é uma das marcas fundamentais do direito e ação política europeus, nomeadamente no que se prende com a tomada de decisões. Com base neste princípio, fixado no art. 5 do Tratado da União Europeia (TUE), as instituições europeias estão obrigadas a que as decisões sejam tomadas o mais próximo possível dos cidadãos. Definindo-o, o princípio da subsidiariedade obriga a elucidar o patamar em que a intervenção se fará - europeu, nacional ou local -, nomeadamente no que se relaciona com a aplicação das competências partilhadas, fazendo com que a UE só possa intervir quando a sua ação seja julgada mais eficaz do que a dos estados-membros a nível nacional ou local (cf. UNIÃO EUROPEIA, 2015; PANIZZA, 2018). Sendo indiscutível as suas virtudes, este princípio possui riscos que podem, sobretudo em períodos de crise, permitir desresponsabilizar as instituições europeias e, principalmente, os estados-membros mais poderosos e grandes contribuintes do orçamento comunitário em relação ao destino dos países mais pobres.

${ }^{11}$ Cf. UNIÃO EUROPEIA, 2014
} 
possa contribuir para a redução das desigualdades na saúde, defender os cidadãos das ameaças sanitárias globais que os podem atingir e melhorar a sustentabilidade dos sistemas de saúde nacionais. No Plano Estratégico 2016-2020 [EUROPEAN COMMISSION, 2017], estes objetivos são reafirmados, sem que, porém, em qualquer dos documentos se faça referência à cobertura universal. Apesar de ser bem conhecido que na UE existem quatro países (Bulgária, Chipre, Grécia e Roménia) em que mais de 10\% dos cidadãos continuam sem cobertura regular de serviços de saúde (OECD/EU, 2016), as instituições europeias preferem olhar para a saúde como mais um instrumento para a promoção da competitividade e, ainda que não de modo explícito, da mercantilização. Esta realiza-se, por exemplo, através das parcerias público-privadas, da gestão privada de hospitais e serviços públicos, da contratualização de serviços a empresas privadas e do chamado "turismo médico e de saúde". Este é cada vez mais visto pelos governos de muitos países europeus como um instrumento de captação de recursos financeiros. No entanto, há que reconhecer, em especial no domínio da prevenção e do tratamento da infeção pelo VIH/sida, que a provisão pública, seja através do financiamento, seja através da prestação de cuidados de saúde, nomeadamente em Portugal, continua a desempenhar um papel-chave. Daqui decorre que os prestadores e as seguradoras privados - que no Brasil asseguram os "plano de saúde" - desempenham um papel marginal, aliás por decisão própria, ao colocarem sistematicamente esta infeção fora das coberturas abrangidas pelos seguros de doença.

No campo mais específico da infeção pelo VIH/ sida, uma das primeiras decisões europeias remonta a 1988, quando o Conselho e os representantes dos governos dos estados-membros acordaram, na sua reunião de 31 de maio desse ano, em sublinhar a importância de uma abordagem comum. Destacando os princípios mais relevantes fixados no documento, em particular nas conclusões, a infeção pelo VIH/ sida é entendida como um problema de saúde pública. Nele se defende também a prioridade absoluta à prevenção através da informação e da educação em matéria de saúde, devendo ser combatida qualquer discriminação ou estigmatização das pessoas afetadas, nomeadamente no que diz respeito ao emprego. É também mencionada a relevância da inclusão dos que trabalham na luta contra a infeção pelo VIH/sida nos programas de intercâmbio de investigadores e de professores (European Communities, 1988). Mais tarde, em 1990, o Conselho de ministros da Saúde reunidos no Conselho de 3 de dezembro de 1990 para avaliar a situação da infeção pelo VIH/sida procura comprometer os estados-membros com estratégias e ações de intervenção baseadas na não-discriminação e não-estigmatização dos indivíduos portadores do vírus, atuando fortemente no campo da toxicodependência, então o grupo mais sujeito a risco de infeção (European Communities, 1990).

Em linha com as responsabilidades e obrigações atribuídas pelos tratados à Comissão Europeia (CE), esta tem mantido especial atenção à infeção pelo VIH/sida. No seu plano de ação para 2014-2016 (EUROPEAN Commission, 2014), enquadrado por um diagnóstico em linha com os realizados pela Onusida, a CE reafirma que a infeção pelo VIH continua a ser um problema da maior relevância para a saúde pública na UE. Convocando o art. ${ }^{\circ} 168^{\circ}$ do TFUE, já acima mencionado, a CE sublinha que, sendo o VIH/sida uma doença transmissível, é necessário que a ação dos governos nacionais seja coordenada a nível europeu. Considerando que a discriminação e a estigmatização estão fortemente presentes na UE, a CE considera que é importante prosseguir com as ações envolvendo os governos nacionais, mas também as organizações não-governamentais (ONG) focadas na defesa dos direitos humanos. Por outro lado, em linha com as principais evidências científicas, a $\mathrm{CE}$ recomenda o tratamento dos doentes desde o diagnóstico da infeção, de forma a diminuir drasticamente os riscos de transmissão. Como grupos prioritários de intervenção, a CE elege os HSH, os utilizadores de drogas injetáveis (UDI) e os migrantes. ${ }^{12}$ Porém, nem uma única vez ao longo do relatório é feita referência às populações mais velhas e, em especial, aos portadores da infeção com mais de cinquenta anos. Dando expressão concreta à "história inexplorada" (Schmid et al., 2009), acima mencionada, a CE, uma das instituiçõeschave da UE, continua a ignorar esta faixa etária, não obstante o seu peso demográfico crescente na infeção pelo VIH/sida. Daqui decorre, de certa forma, uma negligência política em relação aos cidadãos mais idosos que vivem com o VIH/sida. Esta situação tem

\footnotetext{
${ }^{12}$ No livro recente por nós organizado (Sacramento; Ribeiro, 2016), dois capítulos abordam as questões decorrentes das migrações (Bouhdiba, 2016; Hohmann; Olimova, 2016).
} 
implicações na sua saúde e no relacionamento social, colocando mesmo questões relevantes em termos de saúde pública, considerando que um dos problemas que atingem este grupo é, como vimos, o diagnóstico tardio (Lazarus, 2010; UNAIDS, 2014).

Mas a legislação, por mais meticulosa e ajustada que se afigure, por si só não basta. Mesmo depois de quadros políticos bem delineados e a priori adequados, importa avaliar em que medida as disposições políticas vão além da mera discursividade e são consequentes (Sacramento, Ribeiro e Maia 2016), traduzindo-se em instrumentos e formas de intervenção eficazes a nível da prevenção (v.g. campanhas específicas de informação e de sensibilização), da deteção precoce da infeção e dos apoios sociais aos mais idosos, em especial aos que, de entre eles, enfrentam situações de maior vulnerabilidade social. É que a intervenção social no campo do VIH entre pessoas idosas debate-se, amiúde, com constrangimentos bastante severos, desde logo com aqueles que remetem para os estigmas, como nota a National Association of Social Workers (NASW, s.d.) dos EUA:

Os adultos mais velhos são membros de comunidades que são cada vez mais afetadas e infectadas pelo HIV/AIDS, como por exemplo, comunidades alvo de preconceitos raciais, populações mais pobres e homens que fazem sexo com homens. Ser uma pessoa idosa com HIV implica, frequentemente, lidar com o estigma e a discriminação associados ao sexismo, ao classismo, à homofobia e ao racismo.

Ou seja, envelhecer com a infeção amplifica os efeitos da estigmatização, tornando a doença ainda mais excecional para os idosos, já frequentemente sujeitos ao "idadismo" (ageism). Trata-se de um estigma que Butler fez referência pela primeira vez e que definiu como um "processo de estereotipagem sistemática e discriminação contra as pessoas porque elas são velhas, assim como o racismo e o sexismo fazem isso para a cor da pele e para o gênero. As pessoas idosas são caracterizadas como senis, rígidas em pensamentos e práticas, e antiquadas em moralidade e habilidades" (BUTLER, 1969, p. 243). Os estigmas em torno da doença e do envelhecimento podem coexistir e reforçar-se (Emlet et al., 2015), manifestando-se, simultaneamente, às escalas micro, meso e macro (Iversen; Larsen; Solem, 2009).

Considerando os estereótipos, a exclusão social e o sofrimento a que tendem a estar sujeitas as pessoas mais velhas que vivem com o VIH/sida, justificase uma abordagem reforçada dos estados-membros da UE. Em articulação com as instituições europeias, com destaque para a $\mathrm{CE}$, deverão explorar as possibilidades proporcionadas pelos tratados europeus para encontrar soluções que permitam enfrentar os muitos desafios suscitados pela enfermidade, fazendo com que os 50+ deixem de ser, definitivamente, os "unserved, unseen, and unheard" da epidemia, de que nos falavam Emlet e Poindexter (2004) há cerca de década e meia. A sustentabilidade e eficácia destas soluções será largamente assegurada se elas se inscreverem numa abordagem de direitos humanos que vá além da relativa contingencialidade e fragmentação de medidas políticas funcionais para as potenciais vulnerabilidades associadas ao envelhecimento.

Apesar do caminho já percorrido através de convenções e planos internacionais ${ }^{13}$, o foco continua a estar mais nos objetivos e na produção de respostas de política social do que nos direitos e na consequente formulação de princípios normativos vinculativos (Mégret, 2011). Atendendo a que os idosos "sofrem violações de direitos que são, pelo menos em parte, diferentes das que afetam outras pessoas, tornando os instrumentos e meios existentes inadequados" (Mégret, 2011, p. 41), justifica-se a construção de um instrumento internacional específico vocacionado para a salvaguarda dos seus direitos. Certamente que este seria um passo fundamental para começar a esboçar premissas e abordagens mais integradas, sustentadas e vinculativas aos muitos desafios terapêuticos, sociais e de saúde pública gerados, como vimos, pela relevante prevalência do VIH/sida entre as pessoas idosas.

\section{CONSIDERAÇÕES FINAIS}

Constituindo as pessoas com mais de cinquenta anos que vivem com a infeção um grupo cuja situação era relativamente desconhecida até, pelo menos, meados da década passada, não sendo por isso objeto de interesse, quer das entidades e organizações

\footnotetext{
${ }^{13}$ Destacam-se, à escala global, os planos internacionais de ação sobre o envelhecimento de Viena (1982) e de Madrid (2002), elaborados, respetivamente, no quadro das I e II Assembleia Mundial sobre Envelhecimento da ONU (ONU 1983, 2003). No contexto europeu não há propriamente um instrumento político-jurídico específico para o envelhecimento, embora existam diversos regulamentos, diretivas e recomendações a versar sobre o fenómeno (Veiga, 2014) e se procure assegurar a proteção dos idosos em disposições sucintas que constam de instrumentos "generalistas", como é o caso do artigo $25^{\circ}$ da Carta dos Direitos Fundamentais da União Europeia (UNIÃO EUROPEIA, 2000).
} 
internacionais comprometidas com a luta contra o VIH/sida, quer da investigação científica, a progressiva cronicidade da infeção alterou notoriamente a situação. $\mathrm{O}$ aumento significativo da esperança de vida resultante dos avanços no tratamento da infeção pelo VIH/sida e a importância crescente de novos casos de infeção entre os indivíduos com mais de cinquenta anos faz com que este grupo não possa passar "despercebido" às instituições e a todos os que se dedicam ao estudo do VIH/sida. No contexto europeu, o peso deste grupo é, como vimos, significativo, próximo dos $40 \%$ do total dos que vivem com a doença, e representando quase $20 \%$ dos novos casos de infeção. Atendendo que o mundo caminha, em especial nos países mais afluentes, para uma redução significativa da mortalidade e de novos casos, este grupo irá adquirir nos próximos anos uma relevância estatística e social ainda mais forte.

Não sendo a saúde uma competência europeia, continuando sob a tutela dos estados-membros, coloca a estes desafios relevantes que exigem coordenação supranacional no campo das políticas de diagnóstico, tratamento e apoio social às pessoas com mais de cinquenta anos que vivem com a infeção pelo VIH/ sida. Em linha com o recomendado pela organização não-governamental Gay Men's Health Crisis para os Estados Unidos da América (GMHC, 2010), dever-se-á considerar este grupo como constituindo uma população frágil, atingida por múltiplas patologias, na qual o VIH/sida assume um papel preponderante na estigmatização e na perturbação dos laços sociais, aliás observado também por Shippy e Karpiak (2010). Assim, há que colocar no campo da ação política, como grupo prioritário, os mais velhos, construindo, em diálogo com estes e as suas associações, ações de sensibilização e programas de informação, incluindo os diretamente dirigidos por eles. Ainda que dependente de processos de negociação no quadro do exercício da cidadania e da identificação dos seus principais problemas, as respostas não deverão obliterar os indivíduos que têm contacto heterossexual e os HSH e as novas formas de prevenção, como as diretamente ligadas à profilaxia de pré-exposição (PrEP no acrónimo inglês). Em estreita cooperação com a ação no campo do tratamento - para o qual é essencial o sistema público de saúde de acesso universal e não discriminatório -, o estado deverá mobilizar os seus recursos no domínio da intervenção social, identificando e minimizando os efeitos das vulnerabilidades mais graves que afetam os indivíduos infetados, sejam homens, mulheres ou transgéneros.

Socialmente muito diverso, comportando "o viver com HIV/aids" múltiplos sentidos (Valle, 2016), dever-se-á olhar com particular atenção para os mais vulneráveis. A saber, são aqueles que se encontram em situação de desemprego ou subemprego precário, em regra associado a baixos salários, os utilizadores de drogas injetáveis e os que prestam serviços sexuais, melhorando os seus cuidados de saúde e intervindo nos diversos campos sociais que concorrem diretamente para o bem-estar destas pessoas, tais como a alimentação, a habitação e o apoio social às suas tarefas e necessidades quotidianas. Desta forma poder-se-á reduzir os efeitos provocados pela chamada dependência estrutural, especialmente notória, como há muito sublinhou Cabral (2002), entre os idosos pobres em situação de reforma por velhice ou doença. Para tal, como destaca Miller (2010, p. 364), "seria benéfico ter um conjunto internacional de normas para a prevenção do abuso de idosos, desenvolvido como parte de um tratado internacional vinculativo que contemplasse a proteção das suas necessidades e direitos específicos", à semelhança do que já acontece para outros grupos populacionais (v.g., mulheres e crianças) considerados como particularmente sujeitos a vulnerabilidades e constrangimentos.

É atinente referir que, como é sublinhado pelo relatório da Unaids (2013) HIV and aging: a special supplement to the UNAIDS report on the global AIDS epidemic, as pessoas que vivem com o VIH/sida enfrentam riscos mais elevados de complicações de saúde decorrentes da toma prolongada de medicamentos antirretrovirais. Conquanto a adesão terapêutica parece ser mais elevada entre os mais velhos, o envelhecimento e o aumento da morbilidade dele decorrente pode ter efeitos na saúde e, em última instância, na esperança de vida. Não se trata só do risco aumentado de doenças cardiovasculares, mas também das depressões decorrentes da estigmatização e da solidão não raro a ela associada. Assim, há que elaborar políticas direcionadas para o acompanhamento específico deste grupo social, dotando os técnicos e outros atores profissionais de competências para uma intervenção médica e social adequada.

Ainda que as políticas de saúde na UE não possam ser replicadas de modo mecânico a outros 
contextos geográficos, porque são outras as condições políticas e económicas, nomeadamente no que se refere à ação do Estado e à disponibilidade de recursos, é relevante sublinhar o papel decisivo da articulação à escala supranacional. Embora manifestamente insuficiente na UE, como vimos, a cooperação à escala europeia (e também global) no desenho e implementação de políticas de luta contra a infeção pelo VIH/sida, em especial destinada às pessoas com mais de cinquenta anos, é extremamente relevante, considerando, entre outros aspetos, a crescente mobilidade da população, incluindo entre diferentes continentes. Esta cooperação é fundamental para concretizar o getting to zero (zero mortes, zero novas infeções, zero estigma) o grande objetivo da comunidade internacional cuja concretização colocará termo à infeção pelo VIH/sida. Apesar de ainda distante da sua concretização, a UE apresenta resultados esperançosos, nomeadamente na redução da mortalidade, incluindo entre as pessoas com mais de cinquenta anos, tornando patente a importância da ação das instituições públicas na luta contra esta infeção.

Em suma, é fundamental uma abordagem integral que tenha sempre em linha de conta os estigmas que resultam da interseção entre VIH/sida e velhice, procurando intervir no sentido de reverter a fragilidade dos laços sociais de muitas das pessoas idosas que se debatem com o VIH (Shippy; Karpiak, 2005) e potenciar a articulação entre os apoios sociais de proximidade formais e informais, tendo sempre presente que uma vida digna, como destaca Nordenfelt (2003), pressupõe sempre a existência e operacionalização de um quadro de direitos fundamentais que garantam de forma efetiva as necessárias práticas de cuidado e proteção. Não existindo políticas europeias para este grupo de doentes e constatadas as suas múltiplas vulnerabilidades, impõe-se a elaboração de políticas de saúde e de proteção social para elas delineadas, considerando as suas circunstâncias de vida, situações de comorbidade, o acesso aos serviços de saúde e os potenciais estigmas associados à idade.

\section{REFERÊNCIAS}

Alencar, R.; Ciosak, S. Aids em idosos: motivos que levam ao diagnóstico tardio. Revista Brasileira de Enfermagem, v., 69, n. 6, p. 1076-1081, 2016.

Banens, M. Les relations sexuelles des seniors vivant avec le VIH. Sexologies, v. 25, n. 3, p. 122-127, 2016.
Bletzer, K. Experiências e representações do VIH entre trabalhadores agrícolas do leste dos Estados Unidos da América. In: Sacramento, O.; Ribeiro, F. B. (Orgs.). Planeta sida: diversidade, políticas e respostas sociais. Vila Nova de Famalicão: Húmus, p. 177-200, 2016.

Bouhdiba, S. Sida e estigmatização: o caso dos refugiados em África. In: de Famalicão: $\overline{\text { Húmus, p. 201-2 }} \overline{16,2016}$. . Vila Nova

Brooks, J. T.; Buchacz, K.; Gebo, K. A.; et al. HIV infection and older Americans: the public health perspective. American Journal of Public Health, vol. 102, n. 8, p. 1516-1526, 2012.

Butler, R. Age-ism: another form of bigotry. The Gerontologist, v. 9, n. 4, p. 243-246, 1969.

Cabral, M. V.; SILVA, P. A. da; Mendes, H. Saúde e doença em Portugal. Lisboa: Imprensa de Ciências Sociais, 2002.

Camoni, L. et al. The continued ageing of people with AIDS in Italy: recent trend from the national AIDS registry. Annali dell'Istituto Superiore di Sanità, v. 50, n. 3, p. 291-297, 2014.

Cardoso, S. et al. Aging with HIV: a practical review, Brazilian Journal of Infectious Diseases, v. 17, n. 4, p. 464479, 2013.

Craveiro, D. Desigualdades sociais na saúde e envelhecimento: contributos teóricos e empíricos. In: Matos, A.; Schouten, M. J. (Orgs.). Saúde: sistemas, mediações e comportamentos. Vila Nova de Famalicão: Húmus, p. 13-28, 2012.

Dumont, G.-F. The aging of populations in Europe or the demographic winter. Débats, v. 96, n. 2, p. 15-23, 2007.

ECDC/WHO Regional Office for Europe. HIV/AIDS surveillance in Europe 2015. Estocolmo: European Centre for Disease Prevention and Control, 2016. Disponível em: $<$ http://ecdc.europa.eu/en/publications/Publications/HIVAIDS-surveillance-Europe-2015.pdf $>$. Acesso em: 18 ago. 2018.

ECDC/WHO Regional Office for Europe. HIV/AIDS surveillance in Europe 2016. Estocolmo: European Centre for Disease Prevention and Control, 2016. Disponível em: $\quad<$ http://www.euro.who.int/ data/assets/pdf file/0007/355570/20171127-Annual_HIV_Report.pdf $>$. Acesso em: 31 ago. 2018.

Emlet, C.; Poindexter, C. Unserved, unseen, and unheard: integrating programs for $\mathrm{HIV}$-infected and HIV-affected older adults. Health Social Work, v. 29, n. 2, p. 86-96, 2004.

et al. The impact of HIV-related stigma on older and younger adults living with HIV disease: does age matter? AIDS Care: Psychological and Socio-medical Aspects of AIDS/HIV, v. 27, n.4, p. 520-528, 2015.

European Committee of the Regions. Public Health. Subsidiarity monitoring in the area of public health. Bruxelas: Comité das Regiões Europeu, 2018. Disponível 
em: <https://portal.cor.europa.eu/subsidiarity/policyareas/ Pages/PublicHealth.aspx>. Acesso em: 23 ago. 2018.

EUROPEAN COMMISSION. Commission Staff Working Document. Action plan on HIV/AIDS in the EU and neighbouring countries: 2014-2016. Bruxelas: Comissão Europeia, 2014. Disponível em: <https://ec.europa.eu/ health/sites/health/files/sti_prevention/docs/ec_hiv_ actionplan_2014_en.pdf $>$. Acesso em: 22 ago. 2018.

EUROPEAN COMMISSION. Strategic Plan 20162020 - Health \& Food Safety. Ref. Ares(2017)6260978 20/12/2017. Bruxelas: Comissão Europeia, 2017. Disponível em: <https://ec.europa.eu/info/sites/info/files/strategic-plan2016-2020-dg-sante_en_0.pdf>. Acesso em: 22 ago. 2018.

European Communities. Conclusions of the Council and the representatives of the Governments of the Member States, meeting within the Council of 31 May 1988 concerning AIDS. Official Journal of the European Union, C 197, 27/07/1988. Disponível em: <https://eur-lex.europa.eu/ legal-content/PT/ALL/?uri=OJ:C:1988:197:TOC $>$. Acesso em: 24 ago. 2018.

European Communities. Conclusions of the Council and of the Ministers for Health, meeting within the Council of 3 December 1990 on AIDS. Official Journal of the European Communities, C 329, 31/12/1990, p. 0021-22. Disponível em: <https://eur-lex.europa.eu/legal-content/EN/ALL/?uri= OJ\%3AC\%3A1990\%3A329\%3ATOC>. Acesso em: 24 ago. 2018.

EUROSTAT. Death due to AIDS (HIV-disease), by sex. Standardised death rate by 100000 inhabitants. 2016. Disponível em: <http://ec.europa.eu/eurostat/tgm/table.do $?$ tab $=$ table \&plugin $=1 \&$ language $=$ en $\&$ p code $=$ tps $00143>$. Acesso em: 14 ago. 2018.

FFMS. Esperança de vida à nascença: total e por sexo. 2016a. Disponível em: <www.pordata.pt/Europa/Esperan $\%$ c3\%a7a+de+vida+\%c3\%a0+nascen $\%$ c3\%a $7 \mathrm{a}+$ total $+\mathrm{e}+$ por +sexo-1260>. Acesso em: 16 ago. 2018.

FFMS. Esperança de vida aos 65 anos: por sexo. 2016 b. Disponível em: <https:/www.pordata.pt/MicroPage.aspx ?DatabaseName $=$ Europa $\&$ MicroName $=$ Esperan $\% \mathrm{C} 3 \% \mathrm{~A} 7$ $\mathrm{a}+\mathrm{de}+\mathrm{vida}+\mathrm{aos}+65+$ anos + por + sexo\&MicroURL $=1262>$. Acesso em: 16 ago. 2018.

GMHC. Growing Older with the Epidemic: HIV and Aging. Nova Iorque: Gay Men's Health Crisis, 2010. Disponível em: <http://www.gmhc.org/files/editor/file/a pa aging10 emb2.pdf>. Acesso em: 20 ago. 2018.

Hohmann, S.; Olimova, S. Os migrantes tajiques e o VIH/ sida na Rússia: representações, riscos e respostas sociais. In: Sacramento, O.; Ribeiro, F. B. (Orgs.). Planeta sida: diversidade, políticas e respostas sociais. Vila Nova de Famalicão: Húmus, p. 151-176, 2016.
Iversen, T.; Larsen, L.; Solem, P. A conceptual analysis of ageism. Nordic Psychology, v. 61, n. 3, p 4-22, 2009.

Lazarus, J. V.; Nielsen, K. K. HIV and people over 50 years old in Europe. HIV Medicine, n. 11, p. 479-481, 2010.

Mégret, F. The human rights of older persons: a growing challenge. Human Rights Law Review, v. 11, n. 1, p. 37-66, 2011.

Mèndes-Leite, R. Uma feminilidade fugidia: das feridas narcisísticas à feminilidade das mulheres seropositivas. In: MAIA M.; Ribeiro, F. B. (Orgs.). VIH-sida: experiência da doença e percursos de cuidados de saúde. Vila Nova de Famalicão: Húmus, p. 87-102, 2015.

Miller, J. International human rights and the elderly. Marque e Elder's Advisor, v. 11, n. 2, p. 343-365, 2010.

Moyer, E.; Hardon, A. A disease unlike any other? Why HIV remains exceptional in the age of treatment. Medical Anthropology, v. 33 n. 4, p. 263-269, 2014.

NASW. The aging of HIV. National Association of Social Workers . Washington, D.C. Disponível em: < https://www. socialworkers.org/LinkClick.aspx?fileticket $=\mathrm{u} 7 \mathrm{moIMQSJo}$ Y\%3D\&portalid=0>. Acesso em: 20 ago. 2018.

Nguyen, N.; Holodniy, M. HIV infection in the elderly. Clinical Intervention in Aging, v. 3, n. 3, p. 453-472, 2008.

Nordenfelt, L. Dignity and the care of the elderly. Medicine, Health Care and Philosophy, v. 6, n. 2, p. 103-110, 2003.

OECD/EU. Health at a Glance-Europe 2016: State of Health in the EU Cycle. Organisation for Economic Co-operation and Development/European Commission, 23/11/2016. Disponível em: <https:/www.oecd.org/els/health-systems/ Health-at-a-Glance-Europe-2016-CHARTSET.pdf $>$. Acesso em: 24 ago. 2018.

O’Grady, C. Estigma e agência na experiência da doença entre mulheres seropositivas em Chennai, na Índia. In: Sacramento, O.; Ribeiro, F. B. (Orgs.). Planeta sida: diversidade, políticas e respostas sociais. Vila Nova de Famalicão: Húmus, p. 217-236, 2016.

ONU. Vienna International Plan of Action on Aging. Nova Iorque: ONU, 1983. Disponível em: <http://www.un.org/en/ events/elderabuse/pdf/vipaa.pdf>. Acesso em: 6 ago. 2018.

ONU, Declaración Politica y Plan de Acción Internacional de Madrid sobre el Envejecimiento. Nova Iorque: ONU, 2003. Disponível em: <http:/www.un.org/esa/socdev/ documents/ageing/MIPAA/political-declaration-sp.pdf>. Acesso em: 6 ago. 2018.

PANIZZA, R. O princípio da subsidiariedade. Fichas técnicas sobre a União Europeia - 2018. Parlamento Europeu. Maio 2018. Disponível em: <http://www.europarl. europa.eu/ftu/pdf/pt/FTU_1.2.2.pdf $>$. Acesso em: 23 ago. 2018 . 
Pelt, J. V. Aging with HIV/AIDS. Social Work Today, v. 12, n. 3, p. 28, 2012. Disponível em: <http://www.socialworktoday. com/archive/051412p28.shtml>. Acesso em: 23 ago. 2018.

Piketty, T. Le capital au XXIe siècle. Paris: Seuil, 2013.

Piot, P.; Cravero, K. As mulheres e a sida. In: Ockrent, C. (Org.). O livro negro da condição das mulheres. Lisboa: Temas e Debates, p. 179-210, 2007.

Ribeiro, F. B.; Sacramento, O. Viver com a infeção: gestão da doença e quotidianos terapêuticos de doentes com VIH/sida no Nordeste de Portugal. Revista Angolana de Sociologia, n. 10, p. 111-130, 2012.

Enfrentar a infeção: experiência social e processos terapêuticos de seropositivos no distrito de Bragança. In: Maia M.; Ribeiro, F. B. (Orgs.). VIH-sida: experiência da doença e percursos de cuidados de saúde. Vila Nova de Famalicão: Húmus, p. 65-85, 2015.

Sacramento, O.; Ribeiro, F. B. (Orgs.). Planeta sida: diversidade, políticas e respostas sociais. Vila Nova de Famalicão: Húmus.

; Maia M. Sida e Estado em Portugal: contornos da epidemia, políticas e intervenção social. In: .p. 105-126, 2016.

Schmid G. P. et al. The unexplored story of HIV and ageing. Bulletin of the World Health Organization, n. 87, p. 162, 2009. $<$ http://www.who.int/bulletin/volumes/87/3/09-064030/ en/>.

Shippy, R.; Karpiak, S. The aging HIV/AIDS population: fragile social networks. Aging \& Mental Health, v. 9, n.3, p. 246-254, 2005.

UNAIDS. Get on the Fast-Track: the life-cycle approach to HIV. Finding solutions for everyone at every stage of life. Genebra: Joint United Nations Programme on HIV/AIDS, 2016a. Disponível em: < http://www.unaids.org/sites/default/ files/media_asset/Get-on-the-Fast-Track_en.pdf $>$. Acesso em: 23 ago. 2018.

UNAIDS. Global aids update. Genebra: Joint United Nations Programme on HIV/AIDS, 2016b. Disponível em: <http:// www.unaids.org/sites/default/files/media_asset/globalAIDS-update-2016_en.pdf $>$. Acesso em: 17 ago. 2018.

UNAIDS. 2017 Global HIV Statistics. Fact sheet - july 2018. Genebra: Joint United Nations Programme on HIV/AIDS, 2018. Disponível em: $<\mathrm{http}$ ://www.unaids.org/sites/default/ files/media_asset/UNAIDS_FactSheet_en.pdf $>$. Acesso em: 17 ago. 2018.

UNAIDS. HIV and aging: a special supplement to the UNAIDS report on the global AIDS epidemic 2013. Genebra: Joint United Nations Programme on HIV/AIDS, 2013. Disponível em: <http://www.unaids.org/sites/default/ files/media_asset/20131101_JC2563_hiv-and-aging_en_0. pdf $>$. Acesso em: 17 ago. 2018.
UNAIDS. People aged 50 years and older: the GAP report. Genebra: Joint United Nations Programme on HIV/AIDS, 2014. Disponível em: <http://www.unaids.org/sites/default/ files/media_asset/1

2_Peopleaged50yearsandolder.pdf $>$. Acesso em: 17 ago. 2018 .

UNAIDS DATA. Joint United Nations Programme on HIV/ AIDS (UNAIDS). Disponível em: http://www.unaids.org/ sites/default/files/media_asset/unaids-data-2018 en.pdf. Acesso em: 29 ago. 2018.

UNIÃO EUROPEIA. Carta dos direitos fundamentais da União Europeia. Jornal Oficial das Comunidades Europeias, C 364, 18/12/2000. Disponível em: <http://www.europarl. europa.eu/charter/pdf/text_pt.pdf>. Acesso em: 10 ago. 2018.

UNIÃO EUROPEIA. O princípio da subsidiariedade. 03/09/2015. EUR-Lex - Acesso ao direito da União Europeia. 2015. Disponível em: <http://eur-lex.europa.eu/ legal-content/EN/TXT/?uri=uriserv:ai0017>. Acesso em 23 ago. 2018.

UNIÃO EUROPEIA. Regulamento (EU) N. ${ }^{\circ} 282 / 2014$ do Palamento Europeu e do Concelho de 11 de março de 2014 relativo à criação de um terceiro Programa de ação da União no domínio da saúde (2014-2020) e que revoga a Decisão n. ${ }^{\circ} 1350 / 2007 /$ CE. Jornal Oficial da União Europeia, L 86, 21/03/2014. Disponível em: <https://eur-lex.europa.eu/ legal-content/PT/TXT/PDF/?uri=CELEX:32014R0282\&fro $\mathrm{m}=\mathrm{EN}>$. Acesso em: 24 ago. 2018.

UNIÃO EUROPEIA. Saúde. Disponível em: <https:// europa.eu/european-union/topics/health p $\mathrm{pt}$. Acesso em: 10 ago. 2018.

UNIÃO EUROPEIA.Tratado sobre o Funcionamento da União Europeia (versão consolidada). Jornal Oficial da União Europeia, C 326/1, 26/10/2012. Disponível em: $\quad<$ http://eur-lex.europa.eu/legal-content/PT/ TXT/?uri=celex:12012E004>. Acesso em: 6 ago. 2018.

Valle, C. G. do. Política, identidade e cidadania : a sociogénese e os impasses do ativismo biossocial de HIV/ aids no Brasil. In: Sacramento, O.; Ribeiro, F. B. (Orgs.). Planeta sida: diversidade, políticas e respostas sociais. Vila Nova de Famalicão: Húmus, p. 83-103, 2016.

Veiga, M. Legislação da União Europeia para o envelhecimento. Debater a Europa, n. 11, p. 373-394, 2014. Disponível em: $<\mathrm{http}: / /$ debatereuropa.europe-direct-aveiro. aeva .eu/images/n11/mveiga.pdf $>$. Acesso em: 11 ago. 2018.

Wallach, I. L'expérience du vieillissement chez des femmes et des hommes vivant avec le VIH: un vécu à l'intersection du genre, de l'orientation sexuelle et du parcours relié au VIH. Frontières, n. 251, p. 105-126, 2012. 
et al. Le VIH et le vieillissement au Québec: une recherche qualitative sur les expériences, les difficultés et les besoins des personnes vivant avec le VIH de 50 ans et plus. Montréal: Ministère de la Famille et des Aînés, 2013.

Whiteside, A.; Smith, J. Exceptional epidemics: AIDS still deserves a global response. Globalization and Health, n. 5, p. 15, 2009. Disponível em: <http://www. globalizationandhealth.com/content/5/1/15>. Acesso em: 21 dez. 2015.

WHO. Global health sector strategy on HIV 2016-2021: towards ending aids. Genebra: World Health Organization, 2016. Disponível em: <www.unaids.org>. Acesso em: 06 ago. 2018.

WHO. Countries. Genebra: World Health Organization, 2018. Disponível em: <www.euro.who.int/en/countries>. Acesso em: 22 ago. 2018 